\title{
COVID-19 and kidney transplantation: an Italian Survey and Consensus
}

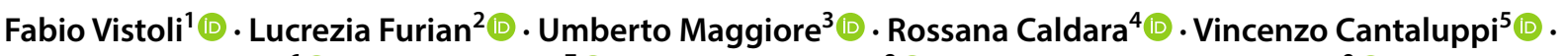

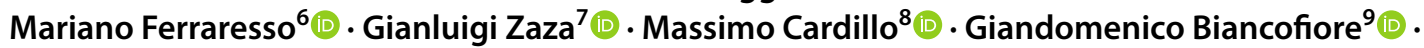 \\ Francesco Menichetti ${ }^{10}$ (D) Alessandro Russo ${ }^{10} \cdot$ Emanuela Turillazzi $^{11}$ (1) . Marco Di Paolo ${ }^{11}$ (1) \\ Giuseppe Grandaliano ${ }^{12} \odot$. Ugo Boggi ${ }^{1} \odot$. on behalf of the Italian National Kidney Transplantation Network - the \\ Joint Committee of the Italian Society of Organ Transplantation and the Italian Society of Nephrology
}

Received: 6 May 2020 / Accepted: 15 May 2020 / Published online: 3 June 2020

(c) Italian Society of Nephrology 2020

\begin{abstract}
Italy was the first Western country to face the COVID-19 pandemic. Here we report the results of a national survey on kidney transplantation activity in February and March 2020, and the results of a three-round Delphi consensus promoted by four scientific societies: the Italian Society of Organ Transplantation, the Italian Society of Nephrology, the Italian Society of Anesthesia and Intensive Care, and the Italian Group on Antimicrobial Stewardship. All 41 Italian transplant centers were invited to express their opinion in the Delphi rounds along with a group of seven experts. The survey revealed that, starting from March 2020, there was a decline in kidney transplantation activity in Italy, especially for living-related transplants. Overall, 60 recipients tested positive for SARS-CoV2 infection, 57 required hospitalization, 17 were admitted to the ICU, and 11 died. The online consensus had high response rates at each round (95.8\%, 95.8\%, and 89.5\%, respectively). Eventually, 27 of 31 proposed statements were approved (87.1\%), 12 at the first or second round (38.7\%), and 3 at the third (9.7\%). Based on the Italian experience, we discuss the reasons for the changes in kidney transplantation activity during the COVID-19 pandemic in Western countries. We also provide working recommendations for the organization and management of kidney transplantation under these conditions.
\end{abstract}

Keywords COVID-19 $\cdot$ Kidney transplantation $\cdot$ Survey $\cdot$ Consensus

\section{Introduction}

SARS-CoV2 is a positive-sense RNA virus belonging to the family of coronaviruses. It causes a flu-like syndrome of varying severity, that is potentially deadly. This disease

Fabio Vistoli and Lucrezia Furian are joint first authors.

Giuseppe Grandaliano and Ugo Boggi are joint senior authors.

The members of Italian National Kidney Transplantation Network are listed in acknowledgement section.

Electronic supplementary material The online version of this article (https://doi.org/10.1007/s40620-020-00755-8) contains supplementary material, which is available to authorized users.

Gianluigi Zaza

gianluigi.zaza@univr.i

Extended author information available on the last page of the article is characterized by one or more of the following symptoms: fever, cough, myalgia, asthenia, dyspnea, sore throat, and diarrhea [1]. The virus was first identified in December 2019 in Wuhan (China). As at April 13, 2020, there were $1,773,084$ cases of confirmed SARS-CoV2 infection worldwide, and 111,652 deaths [2]. In Italy SARS-CoV2 infection was first diagnosed in Rome on January 30, 2020 in two Chinese tourists. The first death caused by the related disease, COVID-19, occurred on February 22, 2020, and involved a 78-year-old man [3]. As at April 13, 2020, SARSCoV2 infection was confirmed in 159,516 patients in Italy, with 20,465 deaths [4].

COVID-19 spreads primarily through contact with respiratory droplets of people infected. In the majority of cases, it only causes mild symptoms and requires no medical intervention [1]. At least $20 \%$ of patients develop a massive and dysregulated inflammatory response, however, which leads to a "capillary leak syndrome" and acute respiratory 
distress syndrome (ARDS). These patients may need ventilatory support and hospitalization in intensive care units (ICUs) [5, 6]. Other complications include shock, acute heart dysfunction, and acute kidney failure. The overall COVID-19 mortality rate is in the range of 3.7-11\% [1].

Reliable information on the rate of SARS-CoV2 infection in uremic patients, be they transplanted [7] or on chronic dialysis [8], are not yet available, but immunosuppression is expected to increase the risk and severity of the infection in kidney transplant recipients.

Italy has universal health coverage insured by a national health system. After the outbreak of COVID-19 in northern parts of the country, the Italian government promptly issued social distancing measures, quickly introducing a country-wide lockdown, and the national health system adopted specific strategies to deal with the epidemic, while protecting both patients and health care personnel. The Civil Protection Department coordinated all these actions [4]. Clinical workflow was revolutionized to prioritize resources for the care of COVID-19 patients, while maintaining acceptable levels of activity for urgent procedures for the general population. Meanwhile, the Italian Transplant Agency (CNT) released recommendations on how to manage transplant programs [9]. As the pandemic spread in other Western countries, their regulatory transplant agencies issued other documents [10].

Despite the tremendous efforts made in the last 2 months to cope with the viral infection worldwide, there is still no objective evidence of the impact of the COVID-19 pandemic on kidney transplantation activities and, more importantly, there is still no guidance on clinical decisions. The aim of this survey and on-line consensus was specifically to provide this missing information.

\section{Methods}

\section{Organizing committee}

The survey and consensus were organized by the joint committee of the Italian Society of Nephrology and the Italian Society of Organ Transplantation.

\section{Participants}

In Italy, kidney transplantation programs $(n=41)$ are part of the public health system, and all the centers involved were invited to participate in this survey. For the consensus, seven experts were also invited to take part in the Delphi rounds. This group of experts consisted of former heads of some of the largest transplant centers in Italy (Enzo Capocasale, Claudio Ponticelli, Silvio Sandrini, Francesco Paolo Schena, Giuseppe Segoloni), a representative of the territorial nephrologists in charge of long-term follow-up (Decenzio Bonucchi), and an expert on Infectious Diseases at the Italian Transplant Agency (Paolo Grossi)".

\section{Discussants}

Consensus results were discussed by experts from the Italian Society of Anesthesia and Intensive Care, and the Italian Group on Antimicrobial Stewardship. The Director of Italian Transplant Agency (CNT), and specialists in Legal Medicine were also asked to discuss the document.

\section{Number of kidney transplants}

Transplant centers were asked to provide figures on kidney transplants performed in the month of February, and between March 1st and March 15th, for the years 2018, 2019, and 2020.

\section{Survey}

An 11-item survey enquired about the practice of kidney transplantation in Italy between equivalent periods of time in 2019 and 2020 (January 1st-March 15th). It was prepared by the joint committee of the Italian Society of Transplantation and Italian Society of Nephrology, and covered the issues raised during remote focus groups held from March 9 to March 11th. The questionnaire also included a space for free comments (Table 1). The survey thus contained physicians' opinions and aggregate data on patients at each transplant center.

\section{Consensus}

The organizing committee identified seven main domains of interest, and defined 31 critical items to address (Tables 2, $3)$. A three-round Delphi consensus $[11,12]$ was then conducted using an online platform made secure by using an academic account (https://docs.google.com/forms). Consensus was defined as at least an $80 \%$ level of agreement. Items not reaching consensus were modified in the light of comments from respondents, then sent back for the next round.

\section{Results}

\section{Survey}

The survey had a $95.1 \%$ response rate (Table 1 ).

Between February 1, and March 15, 2020, a total of 261 kidney transplants were performed. As shown in Fig. 1, there was no reduction in transplantation activity in the month of February compared with the years 2018 and 2019. In the 
Table 1 Italian survey on practice of kidney transplantation during COVID-19 outbreak

\begin{tabular}{|c|c|c|c|}
\hline $\begin{array}{l}\text { 1. Between February 17, } 2020 \text { and March 15, 2020, living-related kidney transplantation } \\
\text { was : }\end{array}$ & $\begin{array}{l}\text { STOPPED } \\
\text { n }(\%) \\
19(55.9 \%)\end{array}$ & $\begin{array}{l}\text { REDUCED } \\
\mathrm{n}(\%) \\
10(29.4 \%)\end{array}$ & $\begin{array}{l}\text { FULLY ACTIVE } \\
\text { n }(\%) \\
5(14.7 \%)\end{array}$ \\
\hline $\begin{array}{l}\text { 2. Between February 17, } 2020 \text { and March 15, 2020, deceased donor kidney transplantation } \\
\text { was }{ }^{\text {b: }}\end{array}$ & $\begin{array}{l}\text { STOPPED } \\
\mathrm{n}(\%) \\
3(7.7 \%)\end{array}$ & $\begin{array}{l}\text { REDUCED } \\
\mathrm{n}(\%) \\
8(20.5 \%)\end{array}$ & $\begin{array}{l}\text { FULLY ACTIVE } \\
\text { n }(\%) \\
28(71.8 \%)\end{array}$ \\
\hline 3. Between February 17, 2020 and March 15, 2020, outpatient follow-up activity was ${ }^{\text {b. }}$ & $\begin{array}{l}\text { STOPPED } \\
\mathrm{n}(\%) \\
2(5.1 \%)\end{array}$ & $\begin{array}{l}\text { REDUCED } \\
\mathrm{n}(\%) \\
35(89.8 \%)\end{array}$ & $\begin{array}{l}\text { FULLY ACTIVE } \\
\mathrm{n}(\%) \\
2(5.1 \%)\end{array}$ \\
\hline $\begin{array}{l}\text { 4. Between February 17, } 2020 \text { and March 15, 2020, enrollment on transplant waiting list } \\
\text { was }{ }^{\text {b: }}\end{array}$ & $\begin{array}{l}\text { STOPPED } \\
\text { n }(\%) \\
18(46.2 \%)\end{array}$ & $\begin{array}{l}\text { REDUCED } \\
\mathrm{n}(\%) \\
14(35.8 \%)\end{array}$ & $\begin{array}{l}\text { FULLY ACTIVE } \\
\text { n }(\%) \\
7(17.9 \%)\end{array}$ \\
\hline $\begin{array}{l}\text { 5. As of March 17, 2020, how many kidney transplant recipients tested positive for COVID- } \\
19 ?^{\text {b }}\end{array}$ & 60 & & \\
\hline 6. How many COVID-19 positive recipients were transplanted in the year 2020 ? & $3(5.0 \%)$ & & \\
\hline 7. As of March 17, 2020, how many COVID-19 positive recipients required hospitalization? ${ }^{\text {b }}$ & $57(95.0 \%)$ & & \\
\hline $\begin{array}{l}\text { 8. As of March 17, 2020, how many COVID-19 positive recipients required Intensive Care } \\
\text { Unit treatment? }\end{array}$ & $17(28.3 \%)$ & & \\
\hline 9. As of March 17, 2020, how many COVID-19 positive recipients have died? ${ }^{\mathrm{b}}$ & $11(18.3 \%)$ & & \\
\hline $\begin{array}{l}\text { 10. As of March } 17,2020 \text {, have you recorded cases of COVID-19 positivity in staff members } \\
\text { caring for transplant recipients? }\end{array}$ & $\begin{array}{l}\text { PHYSICIANS } \\
9\end{array}$ & $\begin{array}{l}\text { NURSES } \\
9\end{array}$ & \\
\hline 11. Do you screen recipients before transplantation for occult SARS-CoV2 infection? & $\begin{array}{l}\text { YES } \\
\text { n (\%) } \\
26(72.2 \%)\end{array}$ & $\begin{array}{l}\text { NO } \\
\text { n (\%) } \\
10(27.8 \%)\end{array}$ & \\
\hline
\end{tabular}

${ }^{\text {a }}$ Response from all the 34 centers with an active live donor program for kidney transplantation

${ }^{\mathrm{b}}$ Response from 39 out 41 (95.1\%) kidney transplant centers

${ }^{\mathrm{c}}$ Response from 36 out 41 (87.8\%) kidney transplant centers

first two weeks of March, 2020, however, there was a clear decline in kidney transplantation activity, especially for living-related procedures (Fig. 1). These figures are reflected in the statements (Table 1). Although more than $70 \%$ of the centers reported having been fully operational for transplantations from deceased donors, several centers admitted having become more selective in accepting donors for kidney transplantation. Modifications to their selection criteria also included a preference for transplantations involving local recipients, though a statement specifically asking about the systematic implementation of this policy was not approved in the Delphi rounds (Table 3).

Only three centers reported having suspended their kidney transplantation program. These programs were hosted in cities in northern Italy, where the incidence of SARS-CoV2 infection was extremely high. Eight other centers only performed transplantation procedures in urgent cases.

Outpatient follow-up activities were reduced at most centers $(89.8 \%)$. In-person visits were warranted only for patients transplanted $<3-6$ months previously, and for medical emergencies. The use of telemedicine was preferred for all other transplant recipients.

Most programs either suspended $(\mathrm{n}=18 ; 46.2 \%)$ or limited $(n=14 ; 35.8 \%)$ visits for the enrolment of patients on the waiting list for a transplant.
Sixty recipients tested positive for SARS-CoV2 infection, including three who were transplanted in February-March, 2020. Fifty-seven recipients (95.0\%) required hospitalization, including 17 who necessitated intensive care (28.3\%), and 11 who died (18.3\%).

Eighteen transplant professionals (9 physicians and 9 nurses) became infected with SARS-CoV2.

Finally, before the Italian Transplant Agency (CNT) made pre-transplant nasopharyngeal swabs (NPhS) mandatory, 26 of 39 centers had already adopted this practice.

\section{Consensus}

The overall response rate was $95.8 \%$ at both the first and the second Delphi round, and $89.5 \%$ at the third.

Out of 31 proposed statements, there was a consensus for 27. Twelve items were agreed at the first or second round (38.7\%), and 3 at the third (9.7\%) (Table 2). Four items were filed without a consensus (12.9\%) (Table 3 ). The consensus was formally divided into seven sets of statements (S). 
Table 2 Agreed statements for kidney transplant practice during COVID-19 pandemic

\begin{tabular}{|c|c|c|c|}
\hline Domain & Statement & Agreement (\%) & Round \\
\hline \multirow[t]{3}{*}{ 1. Kidney transplantation } & $\begin{array}{l}\text { S1.1 During COVID-19 pandemic, live donor transplantation should } \\
\text { be delayed, unless urgent conditions apply }\end{array}$ & 98 & 1 \\
\hline & $\begin{array}{l}\text { S1.2 During COVID-19 pandemic, deceased donor renal transplanta- } \\
\text { tion should be performed only if COVID-19 free pathways can be } \\
\text { ensured }\end{array}$ & 96 & 2 \\
\hline & $\begin{array}{l}\text { S1.3 During COVID-19 pandemic, renal transplantation should } \\
\text { be ensured to immunized patients and to recipients facing urgent } \\
\text { clinical conditions. Individualized decisions should be taken for the } \\
\text { remaining patients }\end{array}$ & 83 & 2 \\
\hline \multirow[t]{2}{*}{ 2. Screening for occult COVID-19 infection } & $\begin{array}{l}\text { S2.1 During COVID-19 pandemic, asymptomatic patients should } \\
\text { be screened by nasopharyngeal swabs before proceeding to renal } \\
\text { transplantation }\end{array}$ & 94 & 1 \\
\hline & $\begin{array}{l}\text { S2.2 During COVID-19 pandemic, asymptomatic patients with } \\
\text { acute rejection should be screened by nasopharyngeal swabs before } \\
\text { administration of anti-rejection treatments }\end{array}$ & 83 & 1 \\
\hline \multirow[t]{6}{*}{ 3. Immunosuppressive therapy } & $\begin{array}{l}\text { S3.1 During COVID-19 pandemic, immunosuppression should be } \\
\text { reduced in asymptomatic patients if nasopharyngeal swab is positive }\end{array}$ & 87 & 1 \\
\hline & $\begin{array}{l}\text { S3.2 During COVID-19 pandemic, immunosuppression should be } \\
\text { reduced in symptomatic patients with positive nasopharyngeal } \\
\text { swabs without clinical or radiologic evidence of lung disease }\end{array}$ & 96 & 1 \\
\hline & $\begin{array}{l}\text { S3.3 During COVID-19 pandemic, immunosuppression should be } \\
\text { reduced in symptomatic patients with positive nasopharyngeal } \\
\text { swabs with clinical or radiologic evidence of lung disease }\end{array}$ & 94 & 1 \\
\hline & $\begin{array}{l}\text { S3.4 During COVID-19 pandemic, in patients with positive naso- } \\
\text { pharyngeal swabs reduction of immunosuppression should be } \\
\text { gauged as follows: } \\
\text { asymptomatic patients: withdraw of anti-proliferative agents; } \\
\text { symptomatic patients without clinical or radiologic evidence of lung } \\
\text { disease: withdraw of anti-proliferative agents and m-TOR inhibitors. } \\
\text { Reduction in the dose of calcineurin inhibitors } \\
\text { symptomatic patients with clinical or radiologic evidence of lung } \\
\text { disease: withdraw of anti-proliferative agents, m-TOR inhibitors, } \\
\text { and calcineurin inhibitors }\end{array}$ & 87 & 2 \\
\hline & $\begin{array}{l}\text { S3.5 During COVID-19 pandemic, in transplant recipients recovering } \\
\text { from COVID-19, infection immunosuppression should be re-intro- } \\
\text { duced 7-15 days after regression of symptoms, if nasopharyngeal } \\
\text { swab is negative and after consultation with a specialist in Infectious } \\
\text { Diseases }\end{array}$ & 85 & 2 \\
\hline & $\begin{array}{l}\text { S3.6 During COVID-19 pandemic, levels of immunosuppression } \\
\text { should be adjusted in patients who receive concurrent antiviral } \\
\text { therapies, after consultation with a specialist in Infectious Diseases } \\
\text { according to possible drug interactions }\end{array}$ & 93 & 1 \\
\hline \multirow[t]{2}{*}{ 4. Other pharmacological agents } & $\begin{array}{l}\text { S4.1 The use of Tocilizumab can be considered in kidney transplant } \\
\text { recipients with severe pneumonia caused by SARS-CoV2 infection }\end{array}$ & 98 & 1 \\
\hline & $\begin{array}{l}\text { S4.2 Steroid boluses can be used in renal transplant recipients with } \\
\text { severe pneumonia caused by SARS-CoV2 infection who need inten- } \\
\text { sive care assistance }\end{array}$ & 91 & 3 \\
\hline
\end{tabular}


Table 2 (continued)

\begin{tabular}{ll}
\hline Domain & Statement \\
\hline 5. Management of kidney transplant recipients & $\begin{array}{l}\text { S5.1 During COVID-19 pandemic, enrollment of patients in the wait } \\
\text { list, for transplants from either deceased or live donors, could be } \\
\text { delayed especially if the transplant center is located in an area with } \\
\text { high prevalence of infection }\end{array}$
\end{tabular}

S5.2 During COVID-19 pandemic, kidney transplant recipients and their cohabitants should strictly adhere to basic protective measures against virus diffusion

S5.3 During COVID-19 pandemic, active transplant programs must offer follow-up visits for patients in the early post-transplant period (3-6 months)

S5.4 During COVID-19 pandemic, kidney transplant outpatients with flu-like symptoms, without dyspnea, should be managed through COVID-19 positive pathways, established for the general population. If hospitalization can be avoided, these recipients should be further assessed remotely. Reduction of immunosuppression could be advised

6. Healthcare professionals

S6.1 During COVID-19 pandemic, cooperative remote recipient surveillance programs should be established by central transplant agencies, to offer post-transplant care to recipients from centers that have become inactive for outpatient services due to logistic or organizational constraints

S6.2 During COVID-19 pandemic, remote expert consultation Agreement (\%) Round

95 3 programs should be established by central transplant agencies, to assist single centers in difficult management decisions for outpatient recipients

S6.3 During COVID-19 pandemic, post-transplant outpatient clinics, in addition to the general safety measures, should include a dedicated space to manage patients with suspected viral infection. Standard safety procedures include surface disinfection after each visit

S6.4 During COVID-19 pandemic, healthcare professionals working in post-transplant outpatient clinics should wear first level personal protection equipment (surgical facemask, disposable isolation gowns and gloves)

S6.5 During COVID-19 pandemic, asymptomatic transplant professionals with positive nasopharyngeal swabs, must be placed on quarantine

S6.6 During COVID-19 pandemic, healthcare professionals taking care of kidney transplant recipients, once positive for COVID-19 on nasopharyngeal swab, should stop their clinical activities and be isolated

7. Assessment of donor

S7.1 During COVID-19 pandemic, live donors who have overcome SARS-CoV2 infection, with a currently negative nasopharyngeal swab, can be considered for donation only after a follow-up period of at least 3 months. In general, live donor renal transplantation should be delayed until the end of the pandemic, unless urgent conditions apply

S7.2 During COVID-19 pandemic, nasopharyngeal swabs should be obtained from all deceased donors

S7.3 During COVID-19 pandemic, nasopharyngeal swabs should be obtained from all live donors in the $24 \mathrm{~h}$ before donation

S7.4 During COVID-19 pandemic, considering that nasopharyngeal swabs may be falsely negative, deep airway swabs should also be obtained from deceased donors 
Table 3 Statements not reaching consensus

\begin{tabular}{|c|c|c|c|c|}
\hline \multirow[t]{2}{*}{ Main topic } & \multirow[t]{2}{*}{ Statement } & \multicolumn{3}{|c|}{$\%$ Agreement } \\
\hline & & $\begin{array}{l}\text { Round } 1 \\
\mathrm{n}=46(\%)\end{array}$ & $\begin{array}{l}\text { Round } 2 \\
\mathrm{n}=46(\%)\end{array}$ & $\begin{array}{l}\text { Round } 3 \\
\mathrm{n}=43(\%)\end{array}$ \\
\hline \multirow[t]{2}{*}{ Kidney transplant activity } & $\begin{array}{l}\text { During COVID-19 pandemic, local graft allocation should be preferred to } \\
\text { reduce patient migration from areas of low-incidence to areas of high- } \\
\text { incidence of infection }\end{array}$ & 59 & 65 & 77 \\
\hline & $\begin{array}{l}\text { During COVID- } 19 \text { pandemic, graft shipping from areas of high-incidence } \\
\text { to areas of low-incidence of infection should be preferred to patient } \\
\text { migration from areas of low-incidence to areas of high-incidence of infec- } \\
\text { tion }\end{array}$ & 59 & 65 & 58 \\
\hline \multirow[t]{2}{*}{ Other pharmacological agents } & $\begin{array}{l}\text { Asymptomatic patients with positive nasopharyngeal swabs should receive } \\
\text { antibiotic prophylaxis }\end{array}$ & 22 & 25 & 23 \\
\hline & $\begin{array}{l}\text { Modification of ACE-inhibitors based anti-hypertensive therapy should } \\
\text { be considered in symptomatic kidney transplant recipients with positive } \\
\text { nasopharyngeal swabs }\end{array}$ & 41 & 63 & 56 \\
\hline
\end{tabular}

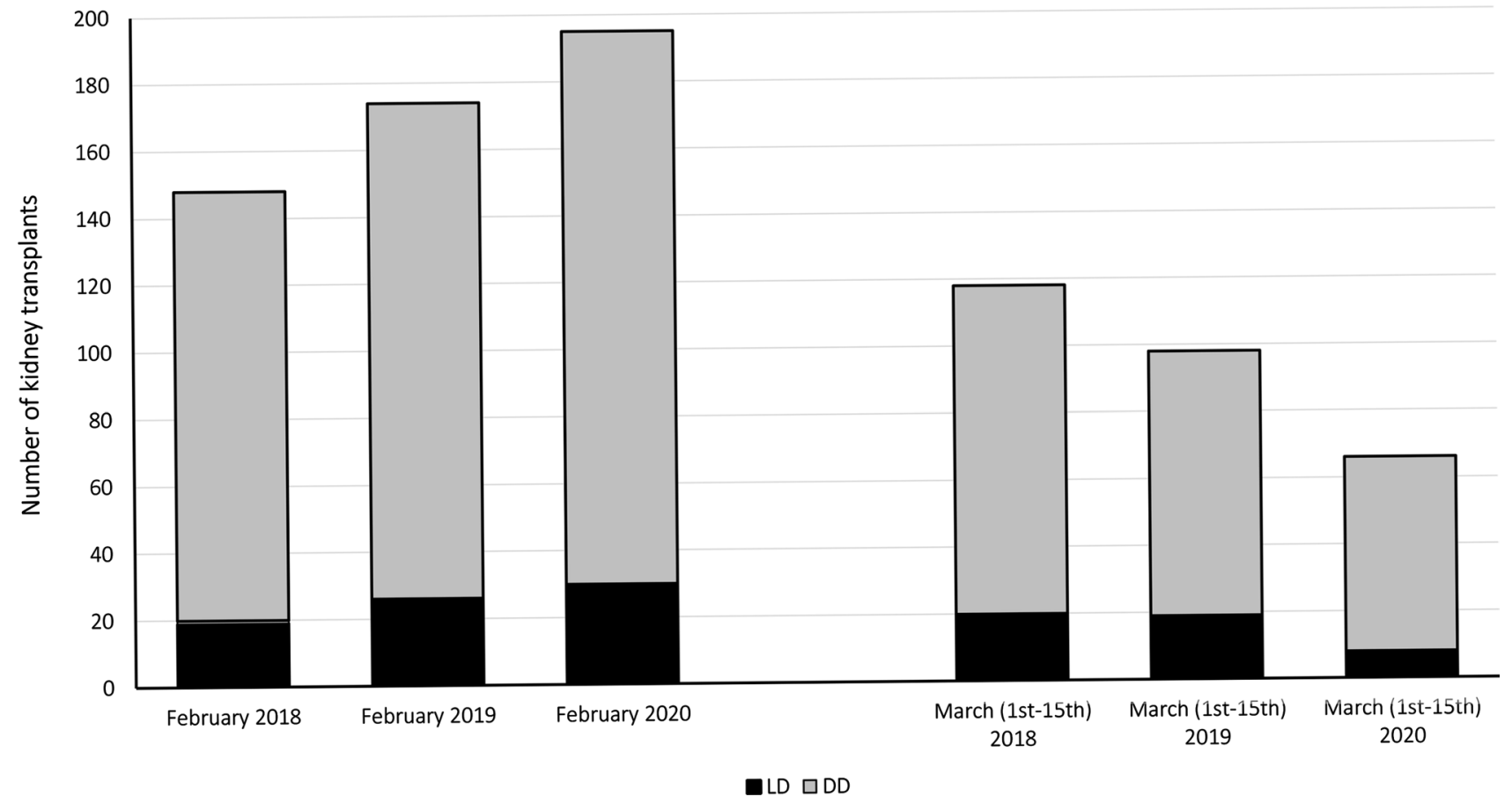

Fig. 1 Number of kidney transplants performed in Italy in the month of February and the first 15 days of March, in three consecutive years (2018-2020). Black bars are living-donor (LD) kidney transplants. Grey bars are deceased-donor (DD) kidney transplants

\section{GROUP 1: Kidney transplant activity}

S.1.1 During the COVID-19 pandemic, live donor transplantation should be delayed unless urgent conditions apply. (Agreement rate 98\%; Delphi round 1).

Comments Urgent conditions only relate to lack of vascular accesses for dialysis and/or unfeasibility of performing peritoneal dialysis. Semi-urgent conditions, such as preemptive recipients wishing to avoid dialysis, are not included in this category because there is no perceived advantage compared with the potential risk of SARSCoV2 infection.

S.1.2 During the COVID-19 pandemic, deceased donor kidney transplantation should be performed only if COVID19 free pathways can be ensured. (Agreement rate 96\%; Delphi round 2).

Comments Besides the availability of COVID-19-free pathways, respondents outlined the importance of the timely 
availability of nasopharyngeal swab, intensive care services, and specifically-trained personnel.

S1.3 During the COVID-19 pandemic, kidney transplantation should be assured for immunized patients and recipients in urgent clinical conditions. Individualized decisions should be taken for other patients. (Agreement rate 83\%; Delphi round 2).

Comments Transplantation should be deemed life-saving for recipients who cannot be maintained on chronic dialysis, and should be delivered accordingly. Transplantation should also be assured for hyperimmunized patients due to the limited chances of finding a compatible donor.

\section{GROUP 2: Nasopharyngeal swabs for COVID}

S2.1 During the COVID-19 pandemic, asymptomatic patients should be screened using nasopharyngeal swabs before proceeding to kidney transplantation. (Agreement rate 94\%; Delphi round 1).

Comments Nasopharyngeal swab detects SARS-CoV2 by means of a reverse-transcriptase-polymerase-chain-reaction (RT-PCR) assay. The method is widely used to screen asymptomatic patients [13]. Its reliability in screening programs is questionable, however, because studies supporting it are retrospective and refer to patients hospitalized with pneumonia. Nasopharyngeal swab may miss recent SARSCoV2 infections [14]. In a population at low risk of infection, nasopharyngeal swabs have a good specificity and a high negative predictive value (approaching 100\%). In Italy, the Italian Transplant Agency (CNT) mandated nasopharyngeal swab in all transplant recipients. A good degree of coordination is needed to ensure that the results of nasopharyngeal swab become available in time.

S2.2 During the COVID-19 pandemic, asymptomatic patients with acute rejection should be screened with nasopharyngeal swabs before any anti-rejection treatments are administered. (Agreement rate 83\%; Delphi round 1).

Comments Infection should be ruled out especially in asymptomatic patients requiring anti-T-cell therapies and/or other vigorous boosts of immunosuppression.

\section{GROUP 3: Immunosuppressive therapy}

S3.1 During the COVID-19 pandemic, immunosuppression should be reduced in asymptomatic patients testing positive on a nasopharyngeal swab. (Agreement rate 87\%; Delphi round 1).

S3.2 During the COVID-19 pandemic, immunosuppression should be reduced in symptomatic patients testing positive on a nasopharyngeal swab but with no clinical or radiological evidence of lung disease. (Agreement rate 96\%; Delphi round 1).

S3.3 During the COVID-19 pandemic, immunosuppression should be reduced in symptomatic patients testing positive on a nasopharyngeal swab and with clinical or radiological evidence of lung disease. (Agreement rate 94\%; Delphi round 1).

S3.4 During the COVID-19 pandemic, the reduction of immunosuppression for patients testing positive on a nasopharyngeal swab should be gauged as follows:

- asymptomatic patients: withdrawal of anti-proliferative agents

- symptomatic patients with no clinical or radiological evidence of lung disease: withdrawal of anti-proliferative agents and m-TOR inhibitors; reduced dosage of calcineurin inhibitors;

- symptomatic patients with clinical or radiological evidence of lung disease: withdrawal of anti-proliferative agents, m-TOR inhibitors, and calcineurin inhibitors. (Agreement rate 87\%; Delphi round 2)

Comments (S3.1-4) Immunological response to infection is reduced in almost all kidney transplant recipients due to long-term administration of immunosuppressants $[15,16]$. Immunosuppression may also significantly influence the clinical presentation and course of viral infections $[17,18]$, that tipically take a severe course in immunocompromised hosts [19]. This would suggest the need to reduce immunosuppression in kidney transplant recipients with SARS-CoV2 infection.

Although a personalized approach is warranted, this consensus provides general recommendations for managing immunosuppression based on severity of SARS-CoV2 infection. Azathioprine and mycophenolic acid/mycophenolate mofetil should be discontinued first because they preferentially inhibit $\mathrm{T}$ cell function and increase susceptibility to viral infections [20]. Although mTOR inhibitors, have some anti-viral effects [21], they should be discontinued next as they can cause various types of lung disease [22] that might complicate the interpretation of radiological and clinical findings in patients at risk of viral pneumonia. While a minimization/discontinuation of calcineurin inhibitors has been associated with an improved course of several viral infections (including CMV, BKV, HHV8 and EBV) [15], these drugs have also been shown to inhibit the proliferation of several coronaviruses in vitro [23-25], so they should be discontinued last.

Low-dose oral corticosteroids, used for maintenance should not be discontinued in order to reduce the risk of acute rejection while preventing the onset of adrenal insufficiency. Steroids may also have a role in controlling 
the dysregulated innate immune response of COVID-19 pneumonia.

S3.5. During the COVID-19 pandemic, immunosuppression should be restored $7-15$ days after symptoms have regressed in transplant recipients recovering from SARSCoV2 infection, providing their nasopharyngeal swabs are negative and subject to consultation with an infectious diseases specialist (Agreement rate 85\%; Delphi round 2).

Comments No clear data exist to suggest the best timing for restoring full immunosuppression after recovery from SARS-CoV2 infection. The threshold given here was established arbitrarily after discussions within the organizing committee and an analysis of comments arriving after the first Delphi round.

S3.6. During the COVID-19 pandemic, immunosuppression levels should be adjusted in patients given concurrent antiviral therapies, after consultation with an infectious diseases specialist regarding possible drug interactions. (Agreement rate 93\%; Delphi round 1).

Comments Antiviral agents are known to interact with immunosuppressants [26, 27]. Levels of immunosuppression should be tailored accordingly.

\section{GROUP 4: Other pharmacological agents}

S4.1. The use of tocilizumab can be considered in kidney transplant recipients with severe pneumonia caused by SARS-CoV2 infection. (Agreement rate 98\%; Delphi round 1).

Comments Tocilizumab is a humanized monoclonal antibody against interleukin-6 receptor (IL-6R), commonly used for rheumatoid arthritis. Tocilizumab has been employed off-label in the treatment of antibody-mediated rejection and, based on recent clinical trials, it appears to be safe in this clinical setting $[28,29]$.

The rationale for using tocilizumab in SARS-CoV2 infection is the clinical relevance of the related inflammatory reaction caused by an enhanced release of IL-6, IL-10, and TNF-alpha. This so-called cytokine storm (recently described in patients, in Wuhan) is also characterized by other immunological dysfunctions, such as a decrease in $\mathrm{CD} 4+\mathrm{T}$ and $\mathrm{CD} 8+$ cell counts, and IFN- $\gamma$ production [30]. Blockade of the IL-6/IL-6R pathway may limit lung injury and the triggering of systemic inflammation that can lead to multiple organ failure, including acute kidney graft dysfunction.

An ongoing multicenter, single-arm, open-label, phase 2 study (TOCIVID-19) (NCT04317092) is investigating the efficacy of tocilizumab in patients with COVID-19 pneumonia $(8 \mathrm{mg} / \mathrm{kg}$ every $12 \mathrm{~h}$, with a maximum of $800 \mathrm{mg}$ per dose) [31]. Transplant recipients cannot be enrolled in this study, however, because previous immunosuppression is one of the exclusion criteria.

S4.2. Steroid boluses can be used in kidney transplant recipients with severe pneumonia caused by SARS-CoV2 infection in need of intensive care. (Agreement rate 91\%; Delphi round 3).

Comments Despite conflicting evidence [32], steroids could be beneficial in treating the hyperinflammation associated with COVID-19 pneumonia [33]. The decision to use steroids should be shared with the intensive care providers responsible for these critically-ill patients because timing [34] and dosage [35] of the treatment are important factors to maximize patients' chances of survival. In transplant recipients the use of steroids is further justified by the concurrent need to reduce/withdraw chronic immunosuppression. Another consideration in favor of using steroids is that the SARS-CoV2 infection of lung alveolar epithelial and endothelial cells has been shown to induce a maladaptive repair mechanism leading to fibrosis [36]. In this setting, steroids may limit the virus's profibrotic activity and contain lung dysfunction. Steroids are mandatory if tocilizumab is used [37].

Guidelines from the Society of Critical Care Medicine and the European Society of Intensive Care Medicine recommend iv. methylprednisolone $1 \mathrm{mg} / \mathrm{kg} / \mathrm{day}$ in patients with moderate-to-severe forms of ARDS $\left(\mathrm{PaO}_{2} / \mathrm{FiO}_{2}<200\right)$. In a recent multicenter trial, early administration of dexamethasone ( $20 \mathrm{mg}$ once daily on days 1 to 5 , then $10 \mathrm{mg}$ once daily on days 6 to 10) to 277 patients with established moderateto-severe ARDS reduced the duration of their mechanical ventilation and overall mortality [38]. Finally, the Surviving Sepsis Campaign guidelines for treating critically-ill adults with COVID-19 recommend using steroids in ARDS patients [39].

\section{GROUP 5: Management of kidney transplant recipients}

S5.1. During the COVID-19 pandemic, the enrollment of patients on the waiting list for transplants from deceased or living donors could be delayed, especially if the transplant center is in an area with a high prevalence of infection. (Agreement rate 95\%; Delphi round 3).

S5.2. During the COVID-19 pandemic, kidney transplant recipients and members of their household should adhere strictly to basic measures to prevent the virus's diffusion. (Agreement rate 100\%; Delphi round 2).

S5.3. During the COVID-19 pandemic, active transplant programs should offer follow-up visits for patients in the early post-transplant period (3-6 months). (Agreement rate 91\%; Delphi round 2). 
S5.4. During the COVID-19 pandemic, kidney transplant outpatients with flu-like symptoms, but no dyspnea, should be managed through pathways established for COVID19-positive cases in the general population. If hospitalization can be avoided, these kidney transplant recipients should continue to be assessed remotely. A reduction of their immunosuppression could be recommended. (Agreement rate 91\%; Delphi round 3).

Comments (S5.1-4) This set of statements indicates that preventive and protective measures applied to the general population should be strictly adopted also by transplant recipients. Transplant centers should also be able to assure patient follow-up soon after transplantation (3-6 months). If working conditions prevent this from taking the form of in-person visits, then remote follow-up should be offered.

\section{GROUP 6: Healthcare professionals}

S6.1 During the COVID-19 pandemic, cooperative remote recipient surveillance programs should be established by central transplant agencies, to offer post-transplant care to recipients from centers that have become inactive for outpatient services due to logistic or organizational constraints. (Agreement rate 89\%; Delphi round 1).

S6.2. During the COVID-19 pandemic, remote expert consultation programs should be established by central transplant agencies, to assist single centers in difficult management decisions for outpatient recipients. (Agreement rate 91\%; Delphi round 1).

S6.3. During the COVID-19 pandemic, post-transplant outpatient clinics, in addition to the general safety measures, should allocate a dedicated space for managing patients with a suspected viral infection. Standard safety procedures include surface disinfection after each visit. (Agreement rate 83\%; Delphi round 2).

S6.4. During the COVID-19 pandemic, healthcare professionals working in post-transplant outpatient clinics should wear first-level personal protective equipment (surgical face masks, disposable isolation gowns and gloves). (Agreement rate 96\%; Delphi round 2).

S6.5. During the COVID-19 pandemic, asymptomatic transplant professionals testing positive on nasopharyngeal swab must be quarantined. (Agreement rate 91\%; Delphi round 1$)$.

S6.6. During the COVID-19 pandemic, health care professionals taking care of kidney transplant recipients who test positive for COVID-19 on nasopharyngeal swab, should suspend their clinical activities and be isolated. (Agreement rate 93\%; Delphi round 2).

Comments (56.1-6) In times when resources have to be prioritized, as in this COVID-19 pandemic, most hospi- tals have retained the ability to cope with urgent surgery. Organ transplantation is, by definition, an urgent procedure. Other activities, such as outpatient services, were suspended or drastically reduced because they were considered non-essential and to contain hospital congestion. Reducing the latter services was also thought to make extra human resources free for the care of acutely ill patients [40]. That said, even if transplantation activities had stopped, transplant services are still taking care of many patients in follow-up. Transplant outpatient services should therefore be maintained during COVID-19 pandemic, taking steps to ensure the safety of both health care providers and recipients. Statement S6.1-6 addresses these new and very complex issues. New organizational models are needed, such as inter-institutional cooperation schemes and/or the creation of centralized services for teleconsultation.

As for protecting health care professionals [41], and preventing the nosocomial diffusion of the disease [42], the statements herein are consistent with the general recommendations of the Center for Disease Control and Prevention [43].

\section{GROUP 7: Donor assessments}

S7.1. During the COVID-19 pandemic, living donors who have overcome SARS-CoV2 infection, with a currently negative nasopharyngeal swab, can be considered for donation only after a follow-up period of at least 3 months. In general, living donor kidney transplantation should be delayed until the end of the pandemic, unless urgent conditions apply. (Agreement rate: 93\%. Delphi round 2).

Comments Needless to say, the safety of living donors is of the utmost importance [44]. In most patients, SARS-CoV2 is no longer detectable 10-20 days after its symptoms have disappeared [45, 46], and there have been no reports of direct COVID-19 transmission from donors to transplant recipients, but the time it takes for definite viral clearance is still unknown. Bearing in mind that living donor kidney transplantation is an elective procedure, the consensus group agreed that extra safety measures should be adopted, waiting for at least 3 months after a negative nasopharyngeal swab.

S7.2. During the COVID-19 pandemic, nasopharyngeal swabs should be obtained from all deceased donors. (Agreement rate: $89 \%$. Delphi round 1).

Comments In Italy, the use of nasopharyngeal swab on donors became mandatory soon after the outbreak of COVID-19. The Italian Transplant Agency (CNT) has recently also mandated bronchoalveolar lavage fluid analysis. There is no clear evidence of COVID-19 being transmitted via the blood or transplanted solid organs, but the lack 
of effective antiviral strategies and the need for immunosuppression make it wise for donors to be screened for occult SARS-CoV2 infection. In donors with a recent computed tomography of the chest, their radiological images could also be used to rule out SARS-CoV2 infection [47]. It is worth adding that COVID-19 can cause direct kidney injury [48].

S7.3. During the COVID-19 pandemic, nasopharyngeal swabs should be obtained from all living donors in the $24 \mathrm{~h}$ before donation. (Agreement rate: $87 \%$. Delphi round 1).

Comments Most hospitals have created COVID-19-free pathways. Transplant procedures should be managed in these pathways, making screening for occult SARS-CoV2 infection prior to admission mandatory. Living donors must also follow this pathway.

S7.4. During the COVID-19 pandemic, deep airway swabs should also be obtained from deceased donors because nasopharyngeal swabs may produce false negative results. (Agreement rate: 89\%. Delphi round 2).

Comments The Italian Transplant Agency (CNT) and the Italian Transplantation Society recommend bronchoalveolar lavage fluid analysis in organ donors [49]. The rationale for this additional test is that COVID-19 causes a viral pneumonia so that the virus could be missed in upper airways while being present in the lower respiratory tract. In keeping with this theoretical background, bronchoalveolar lavage fluid analysis is also mandatory in Italy for patients requiring mechanical ventilation before they can be admitted to a COVID-19-free intensive care units. Although pre-operative bronchoalveolar lavage analysis is not feasible in transplant recipients, swabs from deep airways could be obtained at the time of tracheal intubation. The results will not be available until after the transplant but could still allow for "preemptive therapy", if unexpectedly found positive.

\section{Discussion}

Our survey shows that as of March 1, 2020, when the incidence of SARS-CoV2 infection started to rise steeply [4], in Italy kidney transplantation activity has declined, especially for living donor transplantation. Only three centers, all in areas where the incidence of SARS-CoV2 infection was extremely high, completely suspended their activities. Nonetheless, over $80 \%$ of the programs either reduced or stopped the procedures for enrolling patients on the waiting list, and 95\% reduced or stopped outpatient follow-up visits. In short, despite individual and institutional efforts to ensure the continuation of transplantation activities, the COVID-19 pandemic has clearly affected the practice in Italy. The findings of this survey are in keeping with the recommendations of the consensus. While living donor kidney transplantation should be avoided during the COVID-19 pandemic, transplant centers need to take additional, specific precautions to remain active.

The risk of infection is low for kidney donors admitted to dedicated no-COVID19 surgery units and pre-emptive living-donor kidney transplant candidates with good social support who live in areas with a low incidence of SARSCoV2 infection (e.g., in Central and Southern Italy and some other parts of Europe.

Although the ongoing struggle against the virus has not stopped kidney transplantation in Italy, it has limited the number of procedures and posed new challenges for the overall management of transplant programs. Our survey provides a picture of the Italian situation in mid-March. Epidemiological data show that the incidence of SARSCoV2 infection continued to rise in Italy to mid-April [4], possibly making the final scenario even worse than the one described here.

Our survey also showed that, as at March 17, 2020, 60 kidney transplant recipients in Italy were positive for SARS-CoV2 infection. This figure refers to the known incidence of infection in the population of kidney transplant recipients as a whole-which is difficult to quantify, so it should not be used to estimate the incidence of SARS-CoV2 infection in newly transplanted patients. Although several patients may have been missed due to the short follow-up period, and the risk of exposure to COVID-19 gradually increased as the infection spread in Italy, we identified only three cases of infection among 261 patients transplanted between February 1 and March $15,2020(1.1 \%)$. It is worth noting, however, that $95 \%$ of the total infected recipients required hospitalization, $28 \%$ needed ICU support, and $18 \%$ died. These percentages show that SARS-CoV2 infection in kidney transplant recipients is a matter of serious concern, and reinforce the need for the strict implementation of protective measures. Although scarce, preliminary information on the prevalence and outcomes of COVID-19 in this population suggests that kidney-transplant recipients are not at higher risk of acquiring the viral infection. That said, those affected seem to be at particularly high risk of becoming critically ill with COVID-19 due to their chronic immunosuppression and concomitant health issues [50].

Several serological tests have been proposed for measuring SARS-CoV2-specific antibody response and diagnosing past SARS-CoV2 infections (particularly in asymptomatic cases or patients testing negative on nasopharyngeal swab). Identifying the presence or absence of specific antibodies in kidney transplant recipients may be helpful when it comes to making certain important clinical decisions (e.g., timing and mode of treatment for episodes of acute rejection, evaluation of living donation). 
Measuring SARS-CoV2-specific IgG antibody titers can also be useful in epidemiological studies to help clinicians schedule outpatient follow-up visits appropriately and/or to allow for a safe return of patients to work.

These observations, along with the need to adjust immunosuppression levels in infected recipients, pose new questions regarding how to keep kidney transplant recipients informed during the COVID-19 pandemic. Emerging reports concerning the need to minimize or discontinue immunosuppression in the majority of COVID-19-positive patients should be discussed by health care professionals and their patients. A law recently enacted in Italy (Law 219/2017) [51] establishes that every patient has the right to discuss their values, goals and preferences regarding their health care with physicians, and to have the chance to make decisions about their treatment. The law emphasizes the care relationship and the patient's freedom to decide. As patients have demanded a more participatory role in decisions regarding their health care, they should be fully informed about the nature of their disease, its likely outcome, and the risks and benefits of any proposed treatments, and all these issues should be carefully discussed. Since patients should consider the risks and benefits of any treatment before giving their consent, the risks associated with minimizing or discontinuing immunosuppressantspotentially changing the fate of a functioning kidney transplantation-should be clearly explained so that patients can make a well-informed decision. A COVID-19-tailored information process will make it easier for clinicians and patients to make shared decisions, further empowering caregivers and patients in relation to their own health care.

Given the risk of infection by SARS-CoV2, patients on ambulatory hemodialysis could benefit from a preemptive kidney transplantation providing the transplant surgery can be managed at COVID-19-free transplant centers, and only after testing donors and recipients for COVID-19 with nasopharyngeal swab. Patients may also need to be hospitalized for longer than usual to protect them against the higher risk of SARS-CoV2 infection (associated mainly with the use of induction agents and high doses of immunosuppressants).

Our Delphi consensus produced 27 recommendations intended to provide guidance on the practice of kidney transplantation during the COVID-19 pandemic. Questions could not be posed in the PICO format, and recommendations could not be graded [52], due to the lack of relevant literature to date. Rapidly-evolving research may generate this information soon, but the explosive nature of the COVID-19 pandemic makes it necessary to have some guidelines now, even in the current form of an expert opinion.

In short, as already suggested by several reports [53, 54], the COVID-19 pandemic has major implications for kidney transplantation activities, and of kidney transplant management programs. Several recommendations are provided here to help in this difficult process.

Acknowledgements Italian National Kidney Transplantation Network: Giuliano Brunori, Società Italiana di Nefrologia-SIN, U.O. Nefrologia e Dialisi, Ospedale Santa Chiara, Trento, Italy, giuliano.brunori@apss. tn.it; Flavia Petrini, ICU Dept., Chieti University Hospital, Chieti, Italy, flavia.petrini@unich.it3;Franco Valenza, Fondazione IRCCS Istituto Nazionale Dei Tumori di Milano, Anesthesiology Unit, Italy University of Milan, Department of Pathophysiology and Transplantation, Milan, Italy, franco.valenza@unimi.it; Bruna Lavezzo, Anesthesia and Intensive Care Unit 2, A.O.U. Città della Salute e della Scienza, Torino, Italy, brunalavezzo@libero.it; Decenzio Bonucchi, Nephrology and Dialysis, B. Ramazzini Hospital, AUSL Modena, Carpi (MO) Italy, d.bonucchi@ausl.mo.it; Enzo Capocasale, Division of General Surgery and Organ Transplantation, Azienda Ospedaliero-Universitaria di Parma, Parma, Emilia-Romagna, Italy, ecapocasale15@gmail.com; Paolo Grossi, Infectious Diseases Department, University of Insubria, Varese, Italy, paolo.grossi@uninsubria.it; Claudio Ponticelli, Division of Nephrology Ospedale Maggiore, Milano, Italy, ponticelli.claudio@ gmail.com; Silvio Sandrini, AO Spedali Civili, Brescia, Italy, sandrini. silivio@libero.it; Francesco Paolo Schena,Department of Emergency and Organ Transplantation, University of Bari, Bari, Itay, paolo. schena@uniba.it; Giuseppe Segoloni, Department of Medical Sciences, Renal Transplantation Unit 'A. Vercellone', Division of Nephrology Dialysis and Transplantation, Città della Salute e della Scienza Hospital, University of Turin, Turin, Italy, giuseppe.segoloni@unito. it; Luigi Biancone, Renal Transplant Center "A. Vercellone", Nephrology, Dialysis and Renal Transplant Division, "Città della Salute e della Scienza Hospital", Department of Medical Sciences, University of Turin, Torino, Italy, luigi.biancone@unito.it.; Luigino Boschiero, Department of Surgical Sciences, Kidney Transplant Center, University and Hospital Trust of Verona, Verona, Italy, luigino.boschiero@ aovr. veneto.it; Paolo Rigotti, Kidney and Pancreas Transplantation Unit, Department of Surgery, Oncology and Gastroenterology, Padova University Hospital, University of Padova, Padova, Italy, paolo.rigotti@ univr.it; Giorgia Comai, Department of Experimental Diagnostic and Specialty Medicine, University of Bologna Sant'Orsola- Malpighi Hospital, Bologna, Italy; giorgia.comai@ aosp.bo.it; Nicola Bossini, Operative Unit of Nephrology, A.O. Spedali Civili and University of Brescia, Brescia, Italy, bossini-nicola@libero.it; Enrico Minetti, Renal Unit, Niguarda Hospital, Milan, Italy, enrico.minetti@ospedaleniguarda.it; Giuseppe Iaria, Department of Hepatobiliary Surgery and Transplant Unit, Tor Vergata University of Rome, Italy, giuseppeiaria@ hotmail. com; Andrea Ambrosini, Renal Transplant Unit, Azienda Ospedaliera Ospedale di Circolo, Varese, Italy, Andrea.Ambrosini@asst-settelaghi. it; Pier Giorgio Messa, Nephrology, Dialysis and Renal Transplant Unit, Fondazione IRCCS Ca' Granda Ospedale Maggiore Policlinico Milano Italy, Milan, Italy. piergiorgio.messa@unimi.it; Giuliano Boscutti, Nephrology and Dialysis Unit, Department of Internal Medicine, ASUITS, Trieste, Italy, giuliano.boscutti@asufc.sanita.fvg.it; Mario Carmellini, Renal Transplant Center, Siena University Hospital, Siena, Italy, carmellini@unisi.it; Flavia Caputo, UOC Nephrology 2, Dialysis and Transplantation, ARNAS Civico Palermo, Palermo, Italy. flavia. caputo@tin.it; Andrea Ranghino, SOD Nefrologia, Dialisi e Trapianto Rene, AOU Ospedali Riuniti, Ancona, Italy, andrea.ranghino@ unito.it; Paride De Rosa, General Surgery and Kidney Transplantation Unit, "San Giovanni di Dio e Ruggi d'Aragona University Hospital", Salerno. Italy, paride.derosa@sangiovannieruggi.it; Stefano Federico, Department of Public Health, Section of Nephrology and Renal Transplantation, "Federico II” University, Naples, Italy, federico@unina.it; Massimiliano Veroux, Vascular Surgery and Organ Transplant Unit, Department of Surgery, Transplantation and Advanced Technologies GF Ingrassia, University Hospital of Catania, Catania, Italy, veroux@ unict.it; Calogero Cirami, Nephrology Unit, Careggi University 
Hospital, Florence, Italy, ciramil@aou-careggi.toscana.it; Maurizio Nordio, Nephrology and Dialysis Unit, Provincial Hospital, Camposampiero, Padua, Italy, maurizio.nordio@gmail.com; Franco Citterio, Kidney Transplantation Unit, University Hospital A. Gemelli IRCCS, Rome, Italy, Franco.Citterio@ unicatt.it; Gian Benedetto Piredda, UOC Nefrologia, Azienda Ospedaliera Brotzu, Cagliari, Italy, gianbenedettopiredda@aob.it; Renzo Pretagostini, Department of General Surgery “P. Stefanini," Sapienza University, Rome, Italy, renzopretagostini@ hotmail.it; Paolo De Paolis, Department of Nephrology and Transplant Unit, A.O. S. Camillo-Forlanini, Rome, Italy, PDePaolis@ @camilloforlanini.rm.it; Teresa Rampino, Department of Nephrology, Dialysis and Transplantation, Fondazione IRCCS Policlinico San Matteo, and University of Pavia, t.rampino@smatteo.pv.it; Francesco Pisani, General Surgery and Organ Transplantation, S. Salvatore Hospital, L’Aquila, Italy. pisani.f@ libero.it; Gianni Cappelli, Surgical, Medical, and Dental Department of Morphological Sciences, Section of Nephrology, University Hospital of Modena, Modena, Italy, gianni.cappelli@unimore.it; Antonio Secchi, Transplant Medicine, San Raffaele Scientific Institute, Milan, Italy, secchi.antonio@hsr.it; Paola Salis, Department for the Treatment and Study of Abdominal Diseases and Abdominal Transplantation, IRCCS ISMETT (Istituto Mediterraneo per i Trapianti e Terapie ad alta specializzazione) UPMC (University of Pittsburgh Medical Center) Italy, Palermo, Italy, psalis@ ismett.edu; Fiorella Gastaldon, Department of Nephrology Dialysis and Transplantation San Bortolo Hospital, Vicenza, Italy, fiorella.gastaldon@ aulss8.veneto.it; Francesca Mallamaci, CNR-IFC, Clinical Epidemiology and Physiopathology of Renal Diseases and Hypertension, Reggio Calabria, Italy, francesca.mallamaci@ libero.it; Luca Dello Strologo, Department of Nephrology and Dyalisis, Bambino Gesù Children's Hospital IRCCS, Rome, Italy, luca.dellostrologo@opbg.net; Chiara Taglioni, S. C. Nefrologia e Dialisi, Azienda Ospedaliera di Perugia, S. Andrea delle Fratte, Perugia, Italy, chiara.taglioni@ospedale.perugia.it; Papalia Teresa, Kidney and Transplantation Research Center, Department of Nephrology, Dialysis and Transplantation, "Annunziata" Hospital, Cosenza, Italy, teresapapalia@alice.it; Licia Peruzzi, Pediatric Nephrology Unit, Regina Margherita Children's Hospital, Città della Salute e della Scienza di Torino, Turin, Italy, peruzzilicia@gmail. com; Loreto Gesualdo, Nephrology, Dialysis and Transplantation Unit, Department of Emergency and Organ Transplantation, University of Bari Aldo Moro, Bari, Italy, loreto.gesualdo@uniba.it; Eliana Gotti, Unit of Nephrology and Dialysis, Azienda Socio-Sanitaria Territoriale Papa Giovanni XXIII, Bergamo, Italy, egotti@asst-pg23.it; Paolo Feltracco, Dipartimento di Medicina UOC Istituto di Anestesia e Terapia Intensiva Padova, Italy, paolofeltracco@inwind.it; Ernesto Paoletti, Nephrology, Dialysis and Transplantation, University of Genoa and Policlinico San Martino, Genoa, Italy, ernesto.paoletti@hsanmartino.it.

Funding Not applicable.

\section{Compliance with ethical standards}

Conflict of interest The authors declare that they have no conflict of interest.

Ethics approval Not applicable.

Availability of data and material Not applicable.

\section{References}

1. Cao Y, Liu X, Xiong L et al (2020) Imaging and clinical features of patients with 2019 novel Coronavirus SARS-CoV-2: a systematic review and meta-analysis. J Med Virol. https://doi. org/10.1002/jmv. 25822

2. World Health Organization (WHO) (2020) Coronavirus disease (COVID-2019) situation reports. https://www.who.int/ emergencies/diseases/novel-coronavirus-2019/situation-repor ts. Accessed 11 Apr 2020

3. http://www.salute.gov.it/portale/nuovocoronavirus/detta glioContenutiNuovoCoronavirus.jsp?area $=$ nuovoCoronaviru $\mathrm{s} \& \mathrm{id}=5351 \&$ lingua $=$ italiano\&menu=vuoto Accessed $11 \mathrm{Apr}$ 2020

4. http://opendatadpc.maps.arcgis.com/apps/opsdashboard/index .html\#/b0c68bce2cce478eaac82fe38d4138b1. Accessed 13 Apr 2020

5. World Health Organization (WHO). Infection prevention and control of epidemic- and pandemic-prone acute respiratory infections in health care. WHO Guidel 2014:1-156. http://apps. who.int/iris/bitstream/10665/112656/1/9789241507134_eng. pdf?ua $=1$. Accessed 11 Apr 2020

6. Zhou M, Zhang X, Qu J (2020) Coronavirus disease 2019 (COVID-19): a clinical update. Front Med 14(2):126-135

7. Michaels MG, La Hoz RM, Danziger Isakov L et al (2020) Coronavirus disease 2019: implications of emerging infections for transplantation. Am J Transplant. https://doi.org/10.1111/ ajt. 15832

8. Ikizler TA (2020) COVID-19 and dialysis Units: what do we know now and what should we do? Am J Kidney Dis. https:// doi.org/10.1053/j.ajkd.2020.03.008

9. Centro Nazionale Trapianti. 28 Febbraio 2020 Prot. 482/CNT 2020; 2020

10. https://www.notifylibrary.org/background-documents\#SARSCoV-2 Accessed 11 Apr 2020

11. Diamond IR, Grant RC, Feldman BM et al (2014) Defining consensus: a systematic review recommends methodologic criteria for reporting of Delphi studies. J Clin Epidemiol 67(4):401-409

12. Nair R, Aggarwal R, Khanna D (2011) Methods of formal consensus in classification/diagnostic criteria and guideline development. Semin Arthritis Rheum 41(2):95-105

13. Loeffelholz MJ, Tang YW (2020) Laboratory diagnosis of emerging human coronavirus infections-the state of the art. Emerg Microbes Infect 9(1):747-756

14. Winichakoon P, Chaiwarith R, Liwsrisakun C et al (2020) Negative nasopharyngeal and oropharyngeal swab does not rule out COVID-19. J Clin Microbiol 58(5):e00297-20. https://doi. org/10.1128/JCM.00297-20

15. Fishman JA (2007) Infection in solid-organ transplant recipients. N Engl J Med 357(25):2601-2614

16. Zaza G, Leventhal J, Signorini L et al (2019) Effects of antirejection drugs on innate immune cells after kidney transplantation. Front Immunol 10:2978

17. Guillen E, Pineiro GJ, Revuelta I et al (2020) Case report of COVID-19 in a kidney transplant recipient: Does immunosuppression alter the clinical presentation? Am J Transplant. https ://doi.org/10.1111/ajt.15874

18. Shelhamer JH, Toews GB, Masur H et al (1992) NIH conference. Respiratory disease in the immunosuppressed patient. Ann Intern Med 117(5):415-431

19. Englund J (2011) Viral infections in immunocompromised patients. Biol Blood Marrow Transplant. 17(1Suppl):S2-S5

20. Ritter ML, Pirofski L (2009) Mycophenolate mofetil: effects on cellular immune subsets, infectious complications, and antimicrobial activity. Transpl Infect Dis. 11(4):290-297

21. Berger SP, Sommerer C, Witzke O et al (2019) Two-year outcomes in de novo renal transplant recipients receiving everolimus-facilitated calcineurin inhibitor reduction regimen from the TRANSFORM study. Am J Transplant 19(11):3018-3034 
22. Zaza G, Granata S, Tomei P et al (2014) mTOR inhibitors and renal allograft: Yin and Yang. J Nephrol. 27(5):495-506

23. de Wilde AH, Raj VS, Oudshoorn D et al (2013) MERS-coronavirus replication induces severe in vitro cytopathology and is strongly inhibited by cyclosporin A or interferon- $\alpha$ treatment $\mathbf{J}$ Gen Virol. 94(Pt 8):1749-1760

24. Carbajo-Lozoya J, Müller MA, Kallies S et al (2012) Replication of human coronaviruses SARS-CoV, HCoV-NL63 and HCoV$229 \mathrm{E}$ is inhibited by the drug FK506. Virus Res 165(1):112-117

25. Tanaka Y, Sato Y, Osawa S et al (2012) Suppression of feline coronavirus replication in vitro by cyclosporin A. Vet Res 43:41

26. Bartiromo M, Borchi B, Botta A, et al (2020) Threatening drugdrug interaction in a kidney transplant patient with Coronavirus Disease 2019 (COVID-19). Transpl Infect Dis

27. Trofe-Clark J, Lemonovich $\mathrm{T}$ (2013) Interactions between antiinfective agents and immunosuppressants in solid organ transplantation. Am J Transplant 13(Suppl 4):318-326

28. Choi J, Aubert O, Vo A et al (2017) Assessment of tocilizumab (Anti-interleukin-6 receptor monoclonal) as a potential treatment for chronic antibody-mediated rejection and transplant glomerulopathy in HLA-sensitized renal allograft recipients. Am J Transplant 17(9):2381-2389

29. Shin BH, Everly MJ, Zhang $\mathrm{H}$ et al (2020) Impact of tocilizumab (Anti-IL-6R) treatment on immunoglobulins and anti-HLA antibodies in kidney transplant patients with chronic antibody-mediated rejection. Transplantation 104(4):856-863

30. Chen G, Wu D, Guo W et al (2020) Clinical and immunologic features in severe and moderate Coronavirus Disease 2019. J Clin Invest 130(5):2620-2629

31. https://clinicaltrials.gov/ct2/results?cond=\&term=TOCIVID$19 \&$ cntry $=\&$ state $=\&$ city $=\&$ dist $=$. Accessed 11 Apr 2020

32. Mehta P, McAuley DF, Brown M et al (2020) COVID-19: consider cytokine storm syndromes and immunosuppression. Lancet 395(10229): 1033-1034

33. Russell CD, Millar JE, Baillie JK (2020) Clinical evidence does not support corticosteroid treatment for 2019-nCoV lung injury. Lancet 395(10223):473-475

34. Brun-Buisson C, Richard JC, Mercat A et al (2011) Early corticosteroids in severe influenza $\mathrm{A} / \mathrm{H} 1 \mathrm{~N} 1$ pneumonia and acute respiratory distress syndrome. Am J Respir Crit Care Med 183(9):1200-1206

35. Ariani F, Liu K, Jing Z et al (2013) Glucocorticosteroid in treatment of severe pneumonia. Mediators Inflamm 2013:865635

36. Chen JY, Qiao K, Liu F, et al (2020) Lung transplantation as therapeutic option in acute respiratory distress syndrome for COVID19-related pulmonary fibrosis. Chin Med J (Engl)

37. Nicastri E, Petrosillo N, Bartoli TA et al (2020) National Institute for the Infectious Diseases "L. Spallanzani”, IRCCS. Recommendations for COVID-19 clinical management. Infect Dis Rep 12(1):8543

38. Villar J, Ferrando C, Martínez D et al (2020) Dexamethasone treatment for the acute respiratory distress syndrome: a multicentre, randomised controlled trial. Lancet Respir Med. 8(3):267-276

39. Poston JT, Patel BK, Davis AM (2020) Management of critically ill adults With COVID-19. JAMA. https://doi.org/10.1001/ jama.2020.4914
40. Lancaster EM, Sosa JA, Sammann A et al (2020) Rapid response of an academic surgical department to the COVID-19 pandemic: implications for patients, surgeons, and the community. J Am Coll Surg 230(6): 1064-1073

41. Vitacca M, Nava S, Santus P et al (2020) Early consensus management for non-ICU ARF SARS-CoV-2 emergency in Italy: from ward to trenches. Eur Respir J 55(5):2000632. https://doi. org/10.1183/13993003.00632-2020

42. Wang Y, Wang Y, Chen Y et al (2020) Unique epidemiological and clinical features of the emerging 2019 novel coronavirus pneumonia (COVID-19) implicate special control measures. J Med Virol. https://doi.org/10.1002/jmv.25748

43. https://www.cdc.gov/coronavirus/2019-ncov/index.html. Accessed 11 Apr 2020

44. Ratner LE, Sandoval PR (2010) When disaster strikes: death of a living organ donor. Am J Transplant 10(12):2577-2581

45. Chang Mo G, Yuan X et al (2020) Time kinetics of viral clearance and resolution of symptoms in novel coronavirus infection. Am J Respir Crit Care Med 201(9):1150-1152

46. Liu Y, Yan LM, Wan L et al (2020) Viral dynamics in mild and severe cases of COVID-19. Lancet Infect Dis. https://doi. org/10.1016/S1473-3099(20)30232-2

47. Ai T, Yang Z, Hou H et al (2020) Correlation of chest CT and RTPCR testing in coronavirus disease 2019 (COVID-19) in China: a report of 1014 cases. Radiology. https://doi.org/10.1148/radio 1.2020200642

48. Pan X, Xu D, Zhang H et al (2020) Identification of a potential mechanism of acute kidney injury during the COVID-19 outbreak: a study based on single-cell transcriptome analysis. Intensive Care Med. https://doi.org/10.1007/s00134-020-06026-1

49. Guidance on Coronavirus Disease 2019 (COVID-19) for Transplant Clinicians (org/23-tid/tid-news/657-tid-update-and-guidance-on-2019-novel-coronavirus-2019-ncov-for-transplant-idclinicians). Accessed 13 Apr 2020

50. Montagud-Marrahi E, Cofan F, Torregrosa JV et al (2020) Preliminary data on outcomes of SARS-CoV-2 infection in a Spanish single centre cohort of kidney recipients. Am J Transplant. https ://doi.org/10.1111/ajt.15970

51. https://www.gradeworkinggroup.org/ Accessed 11 Apr 2020

52. Grading Tutorial. https://www.uptodate.com/home/grading-tutor ial. Accessed 11 Apr 2020

53. Picillo R, Seman A (2020) Reflections on life in the time of the Coronavirus pandemic, from the viewpoint of two people who have experienced dialysis and kidney transplantation. J Nephrol. 21:1-3. https://doi.org/10.1007/s40620-020-00731-2

54. Piccoli GB (2020) Hospitals as health factories and the coronavirus epidemic. J Nephrol. 33(2):189-191. https://doi.org/10.1007/ s40620-020-00719-y

Publisher's Note Springer Nature remains neutral with regard to jurisdictional claims in published maps and institutional affiliations. 


\section{Affiliations}

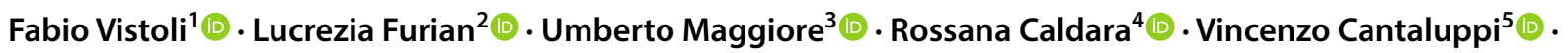

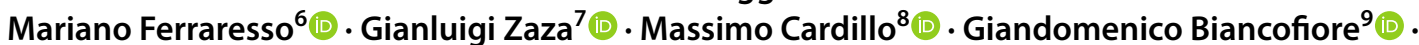

Francesco Menichetti ${ }^{10}$ (1) . Alessandro Russo ${ }^{10}$. Emanuela Turillazzi ${ }^{11}$ (1) $\cdot$ Marco Di Paolo $^{11}$ (1)

Giuseppe Grandaliano ${ }^{12}$ (1) . Ugo Boggi ${ }^{1}$. on behalf of the Italian National Kidney Transplantation Network - the Joint Committee of the Italian Society of Organ Transplantation and the Italian Society of Nephrology

1 Division of General and Transplant Surgery, University of Pisa, Pisa, Italy

2 Kidney and Pancreas Transplantation Unit, Department of Surgical, Oncological and Gastroenterological Sciences, University of Padova, Padua, Italy

3 Kidney and Kidney-Pancreas Transplant Unit, Department of Nephrology, Parma University Hospital, Parma, Italy

4 Department of Internal Medicine, Transplant Medicine Unit, San Raffaele Scientific Institute, Milan, Italy

5 Nephrology and Kidney Transplantation Unit, University of Piemonte Orientale (UPO), Novara, Italy

6 Renal Transplantation, Fondazione IRCCS Ca' Granda Ospedale Maggiore Policlinico, Milan, Italy
7 Renal Unit, Department of Medicine, University Hospital of Verona, Verona, Italy

8 Italian National Transplant Center, Italian National Institute of Health, Rome, Italy

9 Division of Anesthesia and Intensive Care, University of Pisa, Pisa, Italy

10 Division of Infectious Disease Control, University of Pisa, Pisa, Italy

11 Section of Legal Medicine, Department of Surgical, Medical, Molecular Pathology and Critical Area, University of Pisa, Pisa, Italy

12 Division of Nephrology, Università Cattolica del Sacro Cuore, Rome, Italy 